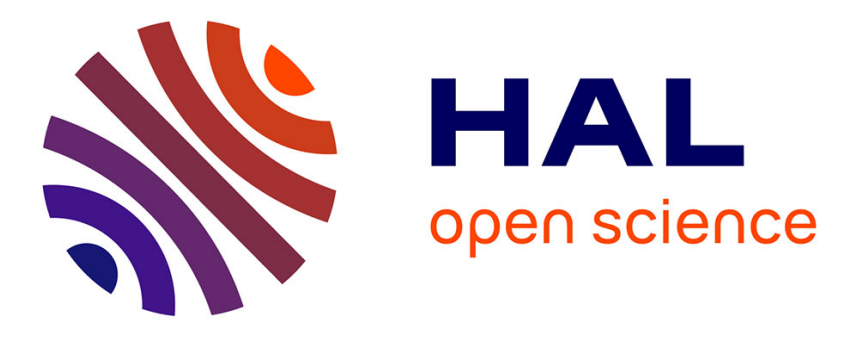

\title{
Recurring models and sensitivity to computational constraints
}

\author{
Anouk Barberousse, Cyrille Imbert
}

\section{To cite this version:}

Anouk Barberousse, Cyrille Imbert. Recurring models and sensitivity to computational constraints. The Monist, 2014, 97 (3), pp.259-279. 10.5840/monist201497318 . hal-01098480

\section{HAL Id: hal-01098480 \\ https://hal.science/hal-01098480}

Submitted on 25 Dec 2014

HAL is a multi-disciplinary open access archive for the deposit and dissemination of scientific research documents, whether they are published or not. The documents may come from teaching and research institutions in France or abroad, or from public or private research centers.
L'archive ouverte pluridisciplinaire HAL, est destinée au dépôt et à la diffusion de documents scientifiques de niveau recherche, publiés ou non, émanant des établissements d'enseignement et de recherche français ou étrangers, des laboratoires publics ou privés. 
Recurring models and sensitivity to computational constraints

(proofs)

$<$ Please use the published version when citing or quoting $>$

Anouk Barberoussse and Cyrille Imbert

Barberousse, Anouk, Cyrille Imbert, « Recurring Models and Sensitivity to Computational

Constraints: » Édité par Sherwood J. B. Sugden. Monist 97, nº 3 (2014): 259-279.

\begin{abstract}
:
Why are some models, like the harmonic oscillator, the Ising model, a few Hamiltonian in quantum mechanics, Poisson equation, or Lokta-Volterra equations, repeatedly used within and across scientific domains, whereas theories allow for much more modeling possibilities? Some historians and philosophers of science have already proposed plausible explanations. For example, Kuhn and Cartwright point to a tendency toward conservatism in science and Humphreys emphasizes the importance of the intractability of what he calls "templates". In this paper, we investigate more systematically the reasons for this remarkable interdisciplinary recurrence. To this aim, we describe in more details the phenomenon we focus on and review competing potential explanations. We disentangle the various assumptions underlying these explanations and propose assessment criteria. Finally, we argue in favor of a specific explanation based on sensitivity to computational constraints and assess its relationships with the other analyzed explanations. -
\end{abstract}

Why are some models repeatedly used within and across scientific domains? Examples of such a striking phenomenon are the harmonic oscillator, the Ising model, a few Hamiltonian in quantum mechanics, Poisson equation, or Lokta-Volterra equations. Even though theories allow for much more modeling possibilities, such models and combinations of them have been incredibly successful in many domains. This phenomenon has already been investigated by a few historians and philosophers of science and is identified as a feature of modeling by scientists.

In this paper, we investigate the reasons for this remarkable interdisciplinary recurrence. Some historians and philosophers of science have already proposed plausible explanations. Kuhn (1996) points to a tendency toward conservatism in periods of normal science. Cartwright (1983) also mentions conservatism and exemplifies the notion in quantum mechanics. Humphreys (2004) introduces the notion of a "template" in order to describe this phenomenon and claims that template intractability plays a major role in its explanation.

Our aim is to analyze these partly competing explanations, as well as other plausible ones, and to argue in favor of the explanation that seems to us the more plausible. To this aim, 
we shall disentangle the various assumptions underlying the competing explanations and propose assessment criteria.

In section 1, we describe in more details the phenomenon we focus on. In section 2, we review potential explanations. Finally, in section 3, we argue in favor of one of these explanations and assess its relationships with the other ones.

\section{Recurring models}

The striking recurrence of a few number of models has been recorded by some physicists, such as Feynman. In the following quote, arguing that physicists only need to master a relatively small part of available mathematics in order to make sense of the physical world, he notes:

"... there is a most remarkable coincidence: The equations for many different physical situations have exactly the same appearance. Of course, the symbols may be different-one letter is substituted for another but the mathematical form of the equations is the same. This means that having studied one subject, we immediately have a great deal of direct and precise knowledge about the solutions of the equations of another." (Feynman, 1965, volume $2, \S 12.1$ )

Throughout this paper, we focus on the "most remarkable coincidence" pointed out by Feynman, that the same equations hold for highly different physical situations.

It can be illustrated by recalling the ubiquity of what is likely to be the best-known recurring model, the harmonic oscillator:

"The classical harmonic oscillator is a model system that has a history of more than a hundred years in the scientific literature and that is discussed in every first-year course in physics. Its has achieved the status of a paradigm because it describes the behavior of a wide variety of systems in physics, chemistry, biology, and other fields. To quote Gitterman [a physicist], "In fact, it has been applied everywhere, from quarks to cosmology ${ }^{1}$." Examples include mechanical systems such as pendula with small angles of displacement and mass-spring systems, acoustical systems and electric systems such as RLC circuits, as well as man-made devices such as clocks and radio circuits." (Méndez et al., 2011; our emphasis)

As mentioned in the introduction, a few historians and philosophers of science have remarked that scientists often draw their modeling tools from a rather limited set. Kuhn goes as far as claiming that this is a major feature of normal science. As is well-known, the whole system of education in science is, according to Kuhn, based on the development of skills allowing students to master a few "exemplars": "The student discovers, with or without the assistance of his instructor, a way to see his problem as like a problem [exemplar] he has already encountered" (1996, 189). Cartwright has insisted that this feature is especially striking in quantum mechanics: students in quantum mechanics first learn how to manipulate a few basic

\footnotetext{
${ }^{1}$ Gitterman, 2013, vii.
} 
Hamiltonians in a few highly idealized situations, and then learn how to adapt these basic Hamiltonians to describe other situations, by combining the already-mastered idealized cases (Cartwright, 1983, 136).

In his 2004 book, Humphreys comments on Feynman's above-quoted observation by emphasizing that it also holds in other disciplines than physics:

"In physics, there is the mathematically convenient fact that three fundamental kinds of partial differential equations - elliptic (e.g., Laplace's equation), parabolic (e.g., the diffusion equation), and hyperbolic (e.g., the wave equation) - are used to model an enormous variety of physical phenomena. This emphasis on a few central models is not restricted to physics. (Humphreys, 2004, 68).

Humphreys adds that the statistician William Feller also remarks that statistical models like the binomial distribution, the normal distribution and the Poisson distribution are used in a surprisingly great variety of problems.

These quotes point to similar phenomena but highlight slightly different aspects. While Kuhn and Cartwright emphasize disciplinary intratheoretical and diachronical recurrence, Humphreys also points at transdisciplinary and potentially synchronical recurrence. Whether these different types of regularities require identical explanations and are of the same type is itself an object of inquiry. To settle this question, or at least make progress about it, a sharper analysis of potential explanations and the hypotheses they rely upon is required. This is what we want to do in the remainder of this paper.

\section{Potential explanations}

In this section, we survey potential explanations from the literature and some others that may come to mind when wondering why such models are repeatedly used despite the variety of modeling practices. We organize these explanations according to their underlying assumptions in order to point out the various images of science they convey.

We first mention a class of explanations that are based on assumptions about nature's regularities. Then we turn to explanations that are based on assumptions about the nature of modeling and the roles of theories and models. Under this heading, we mention a bunch of potential explanations that rely on various conceptions of what it is to be a modeler. At last, we discuss the elements of an explanation that has first been presented by Humphreys (2004). It is based on the assumption that computational features of models play a major role in the explanation of the scarcity of effectively used models in science. The analyses contained in this section will help us argue in favor of the explanation we favor in section 3 .

\subsection{Explanations based on assumptions about nature}

First, one may ground the explanation in a realist interpretation of the models. This type of explanation follows a two-step pattern. First, it assumes that the few effectively used models 
recur because they are efficient in the sense that they yield good predictions. Second, it assumes that the models are efficient because they do capture important features of the investigated phenomena, which share a common abstract structure, so to say. Thus, the models recur because they match actual, recurrent features of phenomena.

2.2 Explanations based on views on the nature and role of scientific theorizing and modeling

Besides explanations based on assumptions about nature, other popular explanations draw on assumptions about the nature on modeling both from the points of view of the models and of the modelers. In this section, we review these explanations by distinguishing four broad categories all pointing toward conservative tendencies. First, there are explanations based on assumptions about the explanatory power of scientific representations, emphasizing the explanatory virtue of relying on just a few models. These economic considerations also govern the second category we focus on, namely explanations based on assumptions about dynamical features of scientific communities. The last two categories draw on assumptions about the (complex) relationships between theories, models, and theoretical languages.

\subsubsection{Explanations based on the explanatory role of theories and models}

The basic assumption underlying this category of explanations is that it is a virtue of a theory to be able to cover a large number of cases via a limited number of mathematical models. As emphasized by Cartwright,

A good theory aims to cover a wide variety of phenomena with as few principles as possible. [...] It is a poor theory that requires a new Hamiltonian for each new physical circumstance. The great explanatory power of quantum mechanics comes from its ability to deploy a small number of well-understood Hamiltonians to cover a broad range of cases, and not from its ability to match each situation one-to-one with a new mathematical representation. That way of proceeding would be crazy. (Cartwright, 1983, 144-5)²

The thrust of this type of explanation is to insist on the unificatory power of practices using a limited pool of models, and, by contrast, on the cognitive dissatisfaction that would result from a situation in which too many models would be used.

Explanations of this type rely on cognitive-economical rules that are supposed to govern the development of scientific theorizing and account for conservative tendencies. They are to be

\footnotetext{
${ }^{2}$ Familiarity with quantum mechanics may suggest an objection against Cartwright's view: in quantum chemistry, for instance, the Hamiltonian is adjusted for each molecule based on specific modeling assumptions, using molecular orbitals, Hartree-Fock models, density functional theory, or some other method; so strictly speaking, different models are being used. However, they can be said to belong to the same family of models and thereby be more explanatory than independent models would because their resolution obeys similar principles. We are grateful to an anonymous reviewer for pointing out this aspect.
} 
distinguished from explanations also relying on economical rules bearing on the functioning of scientific communities that we examine below. For sure, cognitive and economical features are usually entangled, but, as our aim is to shed light on a variety of potential explanatory factors, we present pure versions of them, which correspond to drastically idealized situations.

2.2.2 Explanations based on views on the dynamics of theorizing and modeling within the scientific community

Cognitive-economical factors are usually intertwined with factors related to the economical and social constraints that bear on scientific community. In this section, we put forward two such factors: the requirement of avoiding dispersion of effort and the benefit of coordination.

The explanation based on avoidance of dispersion of effort develops as follows: the ways of studying nature are so numerous that scientific communities need to focus upon just a few in order to avoid waste of cognitive resources. This is so because finding out solutions to scientific problems is time-consuming; it hardly gives immediate results and requires collective, long-term work. To put it in Cartwright's words:

The phenomena to be described are endlessly complex. In order to pursue any collective research, a group must be able to delimit the kinds of models that are even contenders. If there were endlessly many possible ways for a particular research community to hook up phenomena with intellectual constructions, model building would be entirely chaotic, and there would be no consensus of shared problems on which to work. (Cartwright, $1983,143-4)$

Among explanations based on similar considerations, let us also mention that adoption of common standards usually yields huge benefits (even when there is no special reason to adopt one in particular) (De Langhe, forthcoming). This general principle can be applied to model choice. Such an explanation is more general than the one above because it is noncommittal on the specific reasons why the adoption of such or such model is beneficial and on the availability of many ways of representing phenomena.

2.2.3 Explanations based on views on the relationship between theories and models

Let us now turn to explanations that, contrary to the above ones, do not rely on economical considerations but rather on views about the relationship between models and their underlying theories. This relationship can be of at least three different sorts and thus provide modelers with at least three features that may enter in the explanation of why just of few models are effectively used.

First, one may argue that the cognitive content of theories is usually given by a few central models so that using theories implies thinking with these models and using them: "these [models] give content to the theory and these are what beginning students spend the bulk of their 
time learning." (Cartwright $(1983,136)$ The opposed view on the relationship between theories and models is that the content of a theory lies in its principles.

Second, it may also be argued that models and theories often share a common fate. This may in turn be accounted for by the fact that students first learn about theories by a few central models, which, according to Kuhn (cf. Section 1), introduces a significant bias in the choice of subsequent models. We emphasize that these first two features of the relationship between theories and models are partly independent in the sense that the second may obtain without the first being true.

Third, there may still be another sort of privileged relationship between some theories and some types of models: in the context of some theories, some models, be it because of their nature or of the kind of cognitive and epistemic creatures that we are, are more easily amenable to theoretical use than others (cf. Barberousse \& Imbert, 2013). This claim has two versions. According to the negative version, some mathematical models may be less appropriate to carry the content of certain theories; for instance, it may be more difficult for discrete models than for continuous ones to carry the content of continuous theories. Another example is the apparent gap between lattice models, which are anisotropic, and fluid dynamics, whose regularities are isotropic. The positive version has it that conversely, some models may be the natural carriers of the content of their related theory.

According to one's view about the nature of models and their relationship to underlying theories, the focus will be on one aspect or the other. All three aspects are theory- or theory-type relative. Thus, they do not hold in general.

So far, we have reviewed a set of potential explanations of the fact that some models are repeatedly used across the mathematized sciences. Most of them were focused on underlying views on theories and models. As emphasized throughout, these explanations rely on partly competing assumptions. This means that, even if several explanatory factors may sometimes concur, the potential explanations we have reviewed cannot merely be added up and seen as complementary. Moreover, they mostly provide local explanatory factors, holding for specific situations, by contrast with general explanations that would rely on features holding generally. Our task in section 3 will be to assess the relative plausibility and weight of the proposed explanations and to make clear how they can combine. However, the list of potential explanations is still incomplete as we have not yet addressed computational explanations. This we do in the next section.

\subsection{Computational explanations}

Even though some of the above-presented explanations implicitly rely on computational features (for instance, some of them assume that solving models is a difficult task), we find it important to devote an entire section to computational explanations specifically because their scope is much more general than the previous mentioned ones. Moreover, as will appear in this section, they are difficult to capture adequately. 


\subsubsection{Humphreys' emphasis on intractability}

In his book, Humphreys presents elements, which, put together, hint at an explanation that is utterly different from the ones discussed so far. According to him, over and above the various factors mentioned above, modeling activity is governed by the two following principles:

(P1) "it is the invention and deployment of tractable mathematics that drives much progress in the physical sciences". (Humphreys, 2004, 55)

(P2) "most scientific models are specifically tailored to fit, and hence are constrained by, the available mathematics." (Humphreys, 2004, 56)

To put it in a nutshell, according to him, "the scientific landscape in general is shaped by computational constraints." (ibid.)

Thus, Humphreys suggests an explanation in which the computational properties of models constitute the main factors accounting for the fact that some models come over and over in physics.

While agreeing with this computational orientation, we believe that the credentials for the various elements of this explanatory scheme need to be carefully examined, which we shall do now. This will in turn allow us to present our own version of the explanation in terms of computational constraints he points to.

\subsubsection{The effects of computational constraints}

Let us first sketch a computational explanation "à la Humphreys" of the scarcity of models, our purpose being to uncover its underlying assumptions. We emphasize that our reconstruction is at most suggested in Humphreys' book and do not claim that he would accept it in the form we present below.

The starting point of the putative explanatory argument is $(\mathrm{P} 0)$ :

(P0) The models ("theoretical templates") we arrive at by applying general equations like Newton's second law or Schrödinger equation are usually not analytically solvable.

(P0) is highly general in scope: "This inability to draw out analytically solvable templates from theoretical templates is by no means restricted to physics. It is a common phenomenon because most nonlinear ordinary differential equations and almost all partial differential equations have no known analytic solutions." (Humphreys, 2004, 63)

From these observations, it is tempting to derive (P2):

(P2) "Most scientific models are specifically tailored to fit, and hence are constrained by, the available mathematics" (Humphreys, 2004, 56). 
The last step is to claim that from (P0) and (P2), it is possible to infer (CT):

(CT) "The drive for computational tractability underlies the enormous importance of a relatively small number of computational templates in the quantitatively oriented sciences". (Humphreys, 2004, 68)

In the following, we analyze the components of this putative argument, assess the logical relationships among them, and accordingly suggest modifications.

(i) Tractability: objective and epistemic computational landscapes

Let us begin with tractability. (P0) is usually taken to be uncontroversial: as early as 1980, Redhead emphasizes that "science is the art of the soluble" and therefore "of modeling" and simplifying (Redhead, 1980, 162).

Still, there are two aspects to intractability. The first one is intractability per se, that is, the mathematical absence of tractable solutions. The second is intractability-for-scientists, or believed intractability. Accordingly, the objective computational landscape should be distinguished from the epistemic computational landscape ${ }^{3}$. The latter only coincides with the former when results about some problems' intrinsic difficulty have been found, under the form of impossibility results, which are rare and difficult to achieve (cf. Humphreys, 2004, n. 29). However, in the vast majority of cases, we do not know what the objective computational landscape looks like since the complexity of known algorithms merely provide upper bounds to it. Besides, impossibility results usually state that there is no way of solving all the instance of a general target task. This nevertheless leaves open the possibility that particular versions of the task can be performed in some cases. For example, the 3D version of the Ising problem is analytically unsolvable but its 2D version has some analytical solutions (see Mertens, 2002). Matrix inversion has $O\left(\mathrm{n}^{2.373}\right)$ complexity but in some cases, it can be performed more quickly. Typically, sparse matrixes, which are populated primarily with zeros, can have linear complexity (see Press et al, 2002; Tewarson, 1973).

For both computational landscapes the following features are particularly important: first, the difficulty of particular problems; second, the scarcity of tractable models; finally, the difficulty of identifying the latter. These features are partly independent of each other, as there may be plenty of tractable problems that are very difficult to discover, in the same way as it is difficult to find out (and authenticate) random binary strings even if the vast majority of strings are random.

Accordingly, the epistemic computational landscape has the following form. On the one hand, there are some analytically solvable models that are known as such; on the other, some models that are known to be intractable per se. The remaining models -- the vast majority of

${ }^{3}$ By "objective" computational landscape, we mean the computational landscape as determined by pure mathematical features, ie, as independent of the modelers' beliefs about it. 
models -- may be intrinsically tractable or not. Scientists do not know for sure, they at best entertain educated beliefs. ${ }^{4}$

Now that we have made explicit what we mean by "computational landscape", we can turn to our first thesis about it: we claim that the explanatory argument above should refer to the epistemic computational landscape. Did analytical solutions exist without scientists being able to find them out, this would probably make no difference with respect to scientists' attitude toward modeling. This is the reason why we introduce (EP0), the epistemic version of (P0):

(EP0) Scientists often do not know how to solve, or provide tractable algorithms to find out the solution of the models they arrive at by applying theories to target systems, nor do they know whether these models are solvable or whether tractable algorithms exist.

Let us point out en passant that an explanation based on the objective (vs. epistemic) computational landscape might be cooked up. It would have the following form: since the computational landscape is mostly intractable, scientists are conservative because they have experimented (and sometimes know) that the landscape is difficult to explore. However, as indicated above, we have but a few results about the objective tractability of models: there is thus no way of assessing the validity of the explanation. Besides, if it were valid, it would not be incompatible with the epistemic explanation we put forward.

\section{(ii) Implications of intractability}

Let us now examine the implications of the above analysis for the putative explanatory argument we discuss in this section. While there seems to be a consequence relation between (P0) and (P2), there is none between (EP0) and (P2): scientists, unless they have objective results about the intractability of a model, are free to try to find new ways of solving it. Even though (P2) may be true, it does not confine empirical scientists to the frontiers of existing mathematics at a given time. They are often mathematically active, which agrees with (P1). Physicists indeed do create mathematical tools and formalisms, discover algorithms, techniques of proofs, statistical tests, or find analytical solutions to mathematical models, because it is crucial for modeling. This sometimes results in revolutionary mathematics (but not necessarily in tractable models). Onsager's solution to the 2D Ising model is a good example of such a revolutionary finding that contributed to the development of a new field (Baxter, 1989). Accordingly, (P2) should be rephrased in terms of how modelers behave:

(EP2) "When designing scientific models, modelers will usually tailor these to fit the existing available mathematics"

As (EP0) does not entail (EP2), it may be asked in which circumstances modelers follow the conservative tendency described in (EP2). Various factors are susceptible to affect this tendency. They come under two main categories, psychological and sociological on the one hand, and mathematical on the other.

\footnotetext{
${ }^{4}$ This point is difficult to assess, as scientists do usually not / do not usually ? report their failures or such negative beliefs in their publications. negative results or failures. We have made our best to gather up and present evidence about scientists' beliefs on model untractability in section 3.2.
} 
Among the psychological and sociological factors, risk-aversion (or, conversely, riskseeking) may affect the propensity of modelers to engage in solving new mathematical problems. A closely related factor is the belief that the community welcomes (or, conversely, is skeptical toward) new approaches. Hints about the community's attitude can be found in journals' editorial strategies or just by observing whether colleagues are prone to discuss novelties. Pressure to produce a high number of papers per year may also negatively affect audacious tendencies, as it may take more time to publish contributions based on novel mathematics.

Mathematical factors are those involving beliefs about the computational landscape and available mathematics in general. For instance, whether existing mathematics are believed to provide sufficiently rich tools for answering modeling questions, or, conversely are viewed as default tools, may reduce, or conversely intensify, conservative tendencies. Beliefs about the likelihood of coming across new solvable problems, or of facing difficult problems, and about the expected fruitfulness of engaging into difficult problem solving may also play a role. Finally, computational factors relate to the scarcity of (human or computational) resources. Most of these factors are highly context-dependent and may vary according to time, discipline, organization of science, and the development of new mathematical knowledge and technology.

Overall, depending on the scenarios, scientists will exhibit more or less conservative tendencies. Thus, defending an explanation of this type implies providing a conservatisminducing scenario, arguing that it agrees with actual science and showing that other explanations are less convincing. This defines our working plan for section 3.

(iii) Implications of conservatism

Although there seems to be a straightforward relation between (EP2) and the scarcity of effectively-used models, there is not, as we shall see below. For sure, if one assumes that the objective computational landscape only contains a few tractable models, the consequence follows, but, as mentioned above, this is an uncertain hypothesis. Here we present some reasons why (EP2) does not entail that effectively used models are scarce.

First, low innovation rate in model building is not necessarily correlated with reduced choice in available models, since it may suffice to slowly fuel a larger and larger pool of models, ending up with significant variety in the set of available models. Second, the explanation of the scarcity of models may not lie in the features of the chosen items but in the procedures of choice (since scientists may be conservative in their use of procedures). For sure, the number of known tractable models matters. If they are a very few, conservatism may explain why many models are effectively used. However, the number of known tractable models is only one aspect of the issue. It may be the case that the available pool is actually large but that the model selection procedures are such that they select again and again the same models in the pool. Similarly, even if the available pool is not that large, these procedures may account for the fact that some specific models are repeatedly selected while other are rarely so. For instance, the techniques of approximations in use may play a crucial role in limiting the number of effectively-used models: when trying to solve a physical problem, it is usually safer to proceed by making use of reliable, well-entrenched techniques of approximation than to devise a entirely new model from scratch. For example, it is common in physics to start the analysis of a system by investigating how it behaves at, or around, its equilibrium point(s). Further, a frequent approximation is to decompose a function as a series of decreasing terms belonging to a family of well-known 
functions, typically polynomials. Accordingly, at an equilibrium point, the linear terms of the energy cancel out and the first terms of the series are quadratic. If the approximation does not go further, then, for conservative forces, this implies that the force is linear and the solution is a harmonic oscillator. For sure, the fact that the harmonic oscillator is a tractable model is crucial. However, the prevalence of some specific approximation methods points to an explanation of the scarcity of effectively used models that is different from an explanation in terms of the actual availability of tractable models, since the use of approximation methods is logically independent of any assumption about the scarcity of other tractable models. There may be many available, tractable models but just a few in actual use because of the prevalence of a few approximation methods.

To put it in a nutshell, the conservative tendencies expressed in (EP2) do not automatically yield scarcity of effectively used models. The latter is thus not related with conservatism in any straightforward way. There are some missing links that we want to put forward. We do so in section 3.

\section{Conclusion of section 2}

In section 2, we have presented a set of potential explanations for the scarcity of effectively used models. These explanations rely on a large variety of factors. At this point, the question arises of whether it is possible to assess the legitimacy of these various explanations, to depart between those that are incompatible, and to order them according to importance or plausibility. In the next section, we discuss assessment criteria and argue in favor of the claim that computational explanations overcome the others.

\section{The computational SCC explanation}

In this section, we first present the explanation we favor. As expected from section 2.3.2, it is a computational explanation. We name it the Sensitivity to Computational Constraints (SCC) explanation since it gives a central role to the way scientists take computational constraints into account. We provide arguments for it in sections 3.2 and 3.3.

\subsection{Statement of the SCC computational explanation}

Following the discussion presented in section 2, we can now present our own version of the computational explanation of the regular use of a limited number of models across disciplines. However, before doing so, we have to clarify a few points about the precise nature of the explanandum and what is accordingly expected from the explanans.

\subsubsection{Methodological caveat}


Our first methodological observation is that it is a different task to explain the recurrence of any specific model A on the one hand (particular explanandum) and the scarcity of a few recurring models on the other (general explanandum). It is likely that the detailed explanation of the recurrence of a specific model A involves several factors, as different uses of A may have different explanations, possibly belonging to the set presented in section 2 . Also, the recurrence of two models model A and B may be explained different factors. Finally, the importance of the various explanatory factors may vary according to the scientific field under consideration. As a result, some degree of explanatory pluralism seems to be in order. Explanatory judgments are thus likely to be context-sensitive and depend on both the epistemic and social organization of the investigated domain. However, we do not want to provide any quantitative explanation, or focus on intra-field features, or even explain the recurrence of any specific model, but rather a general phenomenon, namely the transversal recurrence of a few number of models across fields. The expected explanans is thus bound to have a general scope and it is unlikely that fieldspecific factors can contribute to account for the general explanandum.

\subsubsection{Computational and result pressures}

The heart of the computational explanation we propose is based on the believed difficulty of developing new tractable models. This difficulty is enhanced by the "publish or perish" rule. Let us present these two points in turn. As their detailed discussion has been made in section 2, we shall be content with summarizing their main components.

As we have made clear in section 2, we have it that one major explanans is the global shape of the epistemic computational landscape. This shape is determined by the following features: known tractable models are limited in number; many models and problems are known to be intractable; problems whose intrinsic difficulty is not known are believed to be often intractable; finally, finding new tractable problems is believed to be difficult. In short, modelers experience, in their daily activity, a high computational pressure.

However, computational pressure is not enough to account for our explanandum, for modelers, when deciding to use an already-solved model or try to find a new one, must also assess whether the adventurous strategy is worth it and assess to what extent they can expect to succeed, how costly in terms of resources the two strategies are and which benefits can be expected from both strategies. Besides, they need to take into account the required pace of result production. Therefore, computational pressure is not the only experienced constraint on modelers, as they also experience, more often that not, result pressure: they are bound to produce results at a regular and quick pace, be it because of personal motivation, the pressure of their scientific communities or surrounding society.

Combined with computational pressure, result pressure explains the conservative tendencies put forward by Kuhn or Cartwright. A complete explanation would exhibit an actual mechanism accounting for the specific nature of the involve conservatism: perhaps the conservative behavior corresponds to a conscious rational strategy or it is the tacit application of methodological norms. Since, depending on the context, the actual mechanism at work may vary, 
we do not need to commit to any such particular mechanism. We indeed want the explanation to be general.

In spite of conservative tendencies, physicists are sometimes bold, tackle new mathematical problems; moreover, and as noted above, scarce but regular inputs might result in a large pool of available models. Whatever the proportion of mavericks and their frequency of success, such advances do not seem to be so rich and quick that they would make the repeated use of oscillators, Poisson processes or Ising-derived models peculiarities of outdated scientists. We try to give evidence in section 3.2 that, at least over the last decades, advances in applied mathematics have been slow.

\subsection{Experienced computational pressure}

We first document features of computational pressure as experienced by modelers in order to emphasize its importance. This will allow us to give a more precise description of the epistemic computational landscape that we take as a main component of the computational explanation we put forward.

First, according to modelers involved in the search for new models, the pool of available, tractable models seems to be shallow. As Mertens, a physicist and computer scientist, emphasizes: "some problems in statistical physics have been exactly solved, but the majority of problems has withstand the efforts of generations of mathematicians and physicists" (Mertens, $2002,43)$. His view is confirmed by surveying the main handbooks of mathematical methods for the empirical sciences (for example Baxter, 1989; Fowler, 1997; Bender and Orszag, 1978, to quote prominent ones), which exhibit significant overlaps in the set of displayed models. These applied mathematics handbooks are general, not field-specific; moreover, the content of fieldspecific mathematical handbooks also overlaps with them (see for instance the links between the heat and Black-Scholes equations in finance in Wilmott et al., 1994 and the use of the logistic equation, oscillators and diffusion equations in mathematical biology in Murray, 2002).

Second, finding new solvable models is believed to be difficult; it only happens rarely. In order to illustrate this point, let us briefly present the fate of the Ising model. Ising solved the one-dimension Ising model in 1925 and Onsager the two-dimension model in 1944. These results raised hope for more general ones, but the three-dimension case resisted for decades till results in computational complexity unraveled an unpleasant surprise. Evaluating the partition function (a key step in statistical physics) of the Ising spin glass in three dimensions is NP-hard (Baharona, 1982), which means that no general algorithm can solve the various versions of the Ising model (e.g. for different configurations, sizes, etc.) quickly. The Ising case is by no means an exception. Various problems in statistical mechanics turn out to be intractable. Here is what Welsh writes in his introduction to a paper surveying the complexity of some physical problems:

"The motivation of this paper is to attempt to review and classify the difficulty of a range of problems, arising in the statistical mechanics of physical systems and to which I was introduced by J.M. Hammersley in the early sixties. Their common characteristics at the time were that they all seemed hard and there was little existing mathematical machinery 
which was of much use in dealing with them. Twenty years later the situation has not changed dramatically; there do exist some mathematical techniques which appear to be tools in trade for this area, subadditive functions and transfer matrices for example, but they are still relatively few and despite a great deal of effort the number of exact answers which are known to the many problems posed is extremely small." (Welsh, 1990, our emphasis).

It may be (rightly) objected that the above clues are based on pessimistic arguments. Complexity theory is usually based on a worst-case analysis ${ }^{5}$ and it is not uncommon that problems whose general worst-case version is intractable have more specific, easier-to-solve versions - and after all, when studying particular physical situations, one solvable model that is sufficiently close to the original one is enough. Unfortunately, finding out exactly solvable cases seems to be difficult as well. For instance, Baxter notes that, in the calculation of critical exponents, exactly solving one different but close model would be enough, but even that seems to be too much to ask:

"... if we could solve a model with the same dimensionality and symmetry as a real system, universality asserts that we should obtain the exact critical exponents of the real system. Unfortunately no short-range genuinely three-dimensional model has been solved. The simplest such model is the three-dimensional Ising model [...]: this has been extensively investigated using the series expansion method (Gaunt and Sykes, 1973), but no exact solution obtained." (Baxter, 1982, 12)

Even though the above quotes and our survey of handbooks in applied mathematics are only indications of the computational pressure modelers face on an everyday basis, they make clear that both the scarcity of available, solvable models and the apparent difficulty to find out new solvable models impose severe constraints on modelers' activity. These two factors define the default epistemic computational landscape and shape the decision space when facing new physical problems. They are thus the bottom constituents of any explanation of the scarcity of effectively used models. Above this bottom line, other specific, complementary factors may be added in order to provide a more complete explanation.

\subsection{The scope of the computational explanation}

In this section, we compare the scopes of the computational explanation as expressed in section 3.2 and of the other explanations listed in section 2. We first present two arguments in favor of the generality of the SCC explanation. Then, we contrast this explanation with would-be complete and general explanations.

Our first argument is based on the commonality of mathematics to empirical sciences. Because applied mathematics is a common tool in the empirical sciences, scientists in different

\footnotetext{
${ }^{5}$ Worst-case time complexity describes the longest time an algorithm may take to solve any instance of size $\mathrm{n}$ of a problem.
} 
disciplines will experience similar computational pressures. This amounts to saying that the scope of the computational explanation is much larger than the scopes of the local explanations mentioned in section 2 , for even if result pressure may vary from field to field, conservative tendencies and the recurrence of some tractable models within each field can be expected to be common features of all fields.

The second, complementary argument comes from the general features of model choice. Theories do not determine model choice and, conversely, models, when interpreted differently, are compatible with different theories. Together with computational pressure, this is part of the explanation of why the same few models may be used again and again across scientific disciplines (see section 1). By contrast, most explanations listed in section 2 may explain the intra-disciplinary recurrence of some models, but leave inter-disciplinary recurrence unexplained. The computational explanation presented in section 3.2 thus outcompetes these explanations.

Nevertheless, we insist that our explanatory scheme is far from being a fully-fledged explanation of the scarcity of effectively used models. Some elements of the would-be complete explanation are left blank, either because we do not want to commit about their exact nature, or because they are context-dependent. For instance, it does not purport to explain which specific models are actually used and to which degree they recur. As mentioned above, there may be no general answer to this question, but only explanatory accounts rooted in contextual case-by-case investigations. Thus, when the looked-for explanation is a complete one, the computational explanation does not compete with other explanations but may be combined with them. In a complete explanation, the specific, historical development of each field is likely to play a major role, as reinforcement effects may account for the privilege given to some models in such or such field. For instance, it is common practice to begin the investigation of a new field by playing with some tractable models in order to gain preliminary understanding in a reasonable time. This situation may influence the further development of the field. As claimed above, computational pressure describes the default decision space within which choices are to be made. But which models are preliminary chosen and how the field does develop may, depending on the case, can be accounted for by arbitrary conventions, ease of interpretation, ease of manipulability, or any apparently beneficial feature a model may have.

In brief, the suggestion is that the explanation of the actual use of specific models will be given by a two-step explanatory scheme: the first step describes the computational space that stringently constraints acceptable choices; the second step builds upon other, more specific features to account for the choice of a specific model within the space of acceptable picks.

\subsection{Other large-scope, mathematical explanations}

Other explanations, which also rely on mathematical features, but are significantly different from computational explanations, may be given of the repeated use of some models across scientific fields. Two types of mathematical explanations are worth mentioning: first, explanations related with the nature of the assumptions that are required for justifying the model, and second, explanations in terms of the overall features of approximation techniques. 
It is a common observation that the necessary assumptions to guarantee that a model applies are more or less easy to meet. Now, models with easy applicability conditions are likely to be used more often that models with tricky ones. A typical example is the Poisson distribution discussed by Humphreys $(2004,88-89)$. In this case, what matters is the nature of the hypotheses needed to apply it, which are specific to the modeled situations, whereas explanations in terms of computational constraints do not rely on any such hypotheses but simply on computational features of the equations.

Second, some models are known to be mathematical approximations for a wide range of other mathematical models. Let us mention the Gaussian distribution, of which the central limit theorem guarantees that it is the limit of other distributions. In this case, the explanation of the recurrence of the Gaussian distribution lies in its mathematical relation to other distributions (for a more refined story, see Lyon, forthcoming).

The scope of these mathematical explanations is potentially as large as the scope of the computational explanation. For sure, models whose applicability conditions are weak (like Poisson equation) are promising representations of a wide array of systems. Yet this does not refute our claim that the shape of the epistemic computational landscape is the major general explanatory feature, as having easily-met assumptions is not sufficient for being regularly used: should these models be intractable, they would hardly be used in practice. Actually, models which have weak application conditions (resp. which are mathematical approximations for a wide range of other mathematical models) and are regularly used are usually computationally attractive. For example, Gaussian distribution, Poisson law and the usual distributions have nice properties in terms of analytic solutions or transforms (Fourier or Laplace) ${ }^{6}$. Conversely, models with simple dynamics but which are computationally unattractive, such as the logistic map (the discrete counterpart of the widely-used continuous logistic equation), do not seem to be as successful.

\section{Conclusion}

Our aim in this paper was to investigate into a phenomenon already identified by Kuhn, Cartwright, or Humphreys: the scarcity of effectively used models in mathematized disciplines. In order to do so, we have first reviewed a bunch of potential explanations. By analyzing the assumptions underlying the computational explanation proposed by Humphreys, we have disentangled the logical relationships between its different parts. This has led us to defend our own version of the computational explanation in terms of computational and result pressures. This explanation nicely accounts for the current experience of modelers. Due to its large scope, it outcompetes other more local explanations but cannot account for the contextual use of specific models.

\section{References:}

${ }^{6}$ This is so even though the Gaussian also suffers from computational difficulties when it comes to evaluating the cumulative distribution function. We thank an anonymous reviewer for pointing out this aspect. 
Baharona, Francisco, (1982), "On the computational complexity of Ising spin glass models", Journal of Physics A: Mathematical and General, 15 (10), 3241-3253

Barberousse, Anouk and Imbert, Cyrille, (2013), "New mathematics for old physics: The case of lattice fluids, Studies in History and Philosophy of Modern Physics, 44 (3), 231-241

Baxter, Rodney. J., (1989), Exactly Solved Models in Statistical Mechanics, Academic Press Inc, 1st edition 1982.

Bender Carl M. and Orszag Steven, (1978), A., Advanced Mathematical Methods for Scientists and Engineers: Asymptotic Methods and Perturbation Theory

Cartwright, Nancy, (1983), How the Laws of Physics Lie, Oxford University Press

De Langhe, Rogier, (forthcoming), "A Unified Approach to the Organization of Cognitive Labor", Philosophy of Science

Fowler, A. C., (1997), Mathematical Models in the Applied Sciences, Cambridge University Press, Cambridge Texts in Applied Mathematics

Gitterman Moshe, (2013), The Noisy Oscillator: Random Mass, Frequency, Damping. New Jersey: World Scientific.

Humphreys, Paul, (2004), Extending Ourselves: Computational Science, Empiricism, and Scientific Method, Oxford University Press,

Kuhn, Thomas, (1996), The Structure of Scientific Revolutions, The University of Chicago Press, ( $1^{\text {st }}$ edition, 1962).

Lyon, Aidan, (forthcoming), "Why are Normal Distributions Normal?", The British Journal for the Philosophy of Science.

Vicenç, Méndez, Werner Horsthemke, Pau Mestres, and Daniel Campos, "Instabilities of the harmonic oscillator with fluctuating damping", (2011), Physical review E 84.

Mertens, Stephan, (2002), "Computational Complexity for Physicists", Computing in Science and Engineering 4 (3) 31-47 May/June

Murray, James Dickson, (2002), Mathematical biology. 1 vol. Interdisciplinary applied mathematics, New York.

Press, William H., Teukolsky, Saul A., Vetterling, William T., \& Flannery, Brian P., (2002), Numerical Recipes in $\mathrm{C}++$. Cambridge University Press.

Redhead, Michael, (1980), "Models in Physics", British Journal for the Philosophy of Science 31: 145-163. 
Tewarson, Reginal P., (1973), Sparse matrices. New York: Academic Press.

Welsh, Dominic. J. A., (1990),“The Computational Complexity of Some Classical Problems from Statistical Physics", in Disorder in Physical Systems, 307-321, Clarendon Press

Wilmott, Paul, Dewynne, Jeff and Howison, Sam, (1994), Option Pricing: Mathematical Models and Computation. Oxford: Oxford Financial Press. 